\title{
Impacto de la pandemia sobre el derecho del buen vivir de educación y su accesibilidad
}

Enviado: 23 de febrero de 2021 / Aceptado: 5 de abril de 2021 / Publicado: 12 de julio de 2021

CRISTINA MERCEDES ROSERO MORÁN

Docente, Junta Nacional de Defensa del Artesano, Tulcán-Ecuador,

cris_mrm15@hotmail.com

JAIME RODRIGO CADENA MORILLO

Docente,UNIANDES Sede Tulcán, Ecuador,

ut.jaimecadena@uniandes.edu.ec

ROSA ALICIA REVELO BOLAÑOS

Docente, U. E."San Antonio de Padua” Tulcán, Ecuador,

rosirevelob@yahoo.es

MILTON GUILLERMO GORDÓN MARTÍNEZ

Docente,UNIANDES Sede Tulcán, Ecuador miltongordon@uniandes.edu.ec

DOI 10.24310/IJNE4.1.2021.12020

\section{RESUMEN}

El presente estudio se enfocó en determinar el impacto de la pandemia sobre el acceso al servicio público de educación con base en los derechos del buen vivir establecidos en la Constitución de la República del Ecuador, utilizando la ruta cualitativa-cuantitativa de la investigación, diseño transversal, alcance descriptivo y adoptando como método el análisis. Mediante la aplicación de encuestas a docentes, estudiantes, padres de familia y abogados, se determinó que el impacto del COVID-19 sobre el acceso al servicio público de la educación, va en una escala de alto a muy alto y la vulneración de la educación como derecho humano, según el enfoque del buen vivir siendo comprensión hegemónica del bienestar en un grado que va de alto a muy alto. Como estrategia didáctica se propuso una guía para emprender la transformación de la educación y el mejoramiento del proceso enseñanza-aprendizaje en tiempos de pandemia en la ciudad de Tulcán.

Palabras Clave: Ley Constitucional, educación, didáctica, pandemias, COVID-19.

\section{ABSTRACT}

Impact of the Pandemic on the Good Living Right of Education and its Accessibility

The present study focused on determining the impact of the pandemic on access to the public education service based on the rights of good living stablished in the Republic Constitution, using the qualitative quantitative research route, cross- sectional design, and descriptive scope and adopting analysis as a method. Through the application of surveys to teachers, students, parents and lawyers, it was determined that the impact of COVID-19 on access to the public service of education ranges from high to very low and the violation of education as a right human according to the approach of good living being hegemonic understanding of good living in a degree that goes from high to very high. As a didactic strategy, a guide was proposed to undertake the transformation of education and the improvement of the teaching-learning process in times of pandemic in Tulcan city.

Keywords: Constitutional Law, education, didacticism, pandemics, COVID-19. 


\section{INTRODUCCIÓN}

Frente a los tiempos de pandemia, la educación se ha visto afectada en sentido formal, la presencialidad de la actividad enseñanza-aprendizaje ha sido suspendida hasta una nueva disposición por el decreto de emergencia sanitaria, que potencialmente puede afectar a la comunidad educativa. Sin embargo, el servicio de educación no puede detenerse, ya que constituye un elemento estratégico, en el desarrollo general de la sociedad.

En tal sentido, la realidad de los discentes, en su contacto para el cumplimiento de la labor educativa, se presenta bajo dos contextos: sin acceso a servicios virtuales, que generalmente se presenta en los sectores rurales más apartados del medio; esto hace necesario implementar estrategias asincrónicas que brinden el cumplimiento mínimo de los objetivos educativos. Por otro lado, se encuentran los estudiantes que cuentan con servicios virtuales, a los cuales se puede mediar su proceso formativo con base en materiales educativos digitales como: textos electrónicos, software, videos, páginas web, entre otros recursos, lo cual permite desarrollar actividades sincrónicas y asincrónicas (Aprendizaje, 2017).

La iniciativa de diferentes Estados, para dinamizar el proceso educativo y llevarlo a todos los rincones, ha sido el uso de las TIC, como aliado estratégico para la difusión de los contenidos educativos. A esta modalidad se le ha llamado de diferentes formas, pero con una singular particularidad que se centra alrededor de una educación a distancia y en la cual todos los medios son válidos: televisor, radio, teléfono, computador, Internet, entre otros. Con base en lo anterior, se establece el problema de investigación: ¿Cuál es el impacto de la pandemia sobre el acceso al servicio público de educación con base en los derechos del buen vivir, establecidos en la Constitución de la República en la ciudad de Tulcán?

Considerando que el derecho a la educación es crecientemente reconocido como plataforma de las políticas educativas de los Estados, es un derecho humano que integra un importante corpus al ser un derecho social, económico y cultural promulgado por la Organización de las Naciones Unidas (ONU) como fundamental desde hace sesenta años (Latapí, 2009); organización que a la vez ha desarrollado el esquema de las " $4 \mathrm{~A}$ ”, en donde la base de este estudio investigativo se encuentra inmerso en la accesibilidad cimentando que el derecho a la educación debe estar jurídicamente de manera material y formal para todos con tres dimensiones: la no discriminación, la accesibilidad económica y la accesibilidad material, última que enfoca la posibilidad de efectivizar este derecho aún por la localización geográfica o el uso de tecnología moderna (Scioscioli, 2016). 
En norma supra constitucional el derecho a la educación se encuentra reconocido en la Declaración Universal de Derechos Humanos en el artículo 26, así como en el Pacto Internacional de Derechos Económicos, Sociales y Culturales en los artículos 13 y 14, tratados internacionales de derechos humanos ratificados por el Ecuador (Erazo, 2020).

A nivel constitucional, en Ecuador se tutela este derecho según lo establecen los derechos del buen vivir conforme el artículo 26 de la Constitución de la República del Ecuador: La educación es un derecho de las personas a lo largo de su vida y un deber ineludible e inexcusable del Estado [...] así como el artículo 16.2: todas las personas, en forma individual o colectiva, tienen derecho a: [...] El acceso universal a las tecnologías de información y comunicación y el artículo 17.2: El Estado [...] facilitará [...] el acceso universal a las tecnologías de información y comunicación, en especial para las personas y colectividades que carezcan de dicho acceso o lo tengan de forma limitada; a lo que añade el Régimen del Buen Vivir en el artículo 347.8: será responsabilidad del Estado: [...] incorporar las tecnologías de la información y comunicación en el proceso educativo [...] (Asamblea Constituyente República del Ecuador, 2008).

Los anteriores preceptos doctrinarios y normativos justifican lo imperante del presente estudio investigativo, conjugando lo jurídico con el proceso enseñanza -aprendizaje virtual, que obligó adoptar los tiempos de pandemia en la ciudad de Tulcán.

Para llevar a cabo el presente estudio, se utilizaron los paradigmas cualitativo-cuantitativo de la investigación, con un alcance descriptivo, método analítico y como técnica la encuesta aplicada a docentes, estudiantes, padres de familia y abogados seleccionados con un método de muestreo no probabilístico; orientado a determinar el impacto de la pandemia sobre el acceso al servicio público de educación, con base en los derechos del buen vivir establecidos en la Constitución de la República en la ciudad de Tulcán.

\section{METODOLOGÍA}

La ruta de la investigación empleada fue la cualitativa-cuantitativa, puesto que el impacto de la pandemia sobre el acceso al servicio público de educación se lo estudió en un contexto concreto, con un diseño tipo transversal que permitió la descripción y análisis del impacto de la pandemia en un momento dado. El alcance de la investigación es de tipo descriptivo, puesto que el propósito fue de caracterizar los rasgos importantes de los derechos del buen vivir establecidos en la Constitución de la República (Hernández-Sampieri y Mendoza Torres, 2018). 
El método de muestreo empleado es de tipo no probabilístico y por conveniencia, ya que, por tiempos de pandemia, se seleccionó a un grupo de docentes, estudiantes, padres de familia y abogados de la ciudad de Tulcán, a los cuales se les aplicó una encuesta en Microsoft Word enviada y receptada a través de: correo electrónico, WhatsApp y redes sociales.

Como instrumento de recopilación de información se utilizó el cuestionario con diferentes alternativas de respuesta, escala de valoración y preguntas abiertas. La validación del cuestionario se la realizó a través de una prueba piloto, aplicada a un grupo de integrantes de la comunidad educativa y un especialista en derecho. Esta validación dio confiabilidad, credibilidad y valor al instrumento aplicado.

Los métodos empleados para el procesamiento de la información fueron el analítico-sintético y sistémico, de esta manera, el problema investigado fue dividido en partes, para realizar un estudio individual de sus componentes y los resultados integrados pueden ser generalizados a otros sectores del Ecuador y posiblemente al mundo entero (Gómez Armijos, et al., 2017). Se realiza un análisis por frecuencia relativa, atendiendo al porcentaje de extracto por cada pregunta consultada y en cada cuestionario aplicado.

\section{RESULTADOS}

En la siguiente tabla se muestra los resultados obtenidos de la aplicación de las encuestas a docentes de educación media y superior, con la finalidad de recopilar información sobre el impacto de la pandemia sobre el acceso al servicio público de educación en la ciudad de Tulcán.

Tabla 1. Encuesta aplicada a docentes de educación media y superior. Fuente: elaboración propia

\begin{tabular}{|c|c|c|c|c|c|c|c|c|c|c|c|c|}
\hline \multirow{2}{*}{ DESCRIPCIÓN } & \multicolumn{2}{|l|}{1} & \multicolumn{2}{|l|}{2} & \multicolumn{2}{|l|}{3} & \multicolumn{2}{|l|}{4} & \multicolumn{2}{|l|}{5} & \multicolumn{2}{|c|}{ Total } \\
\hline & $\mathbf{F}$ & $\%$ & $\mathbf{F}$ & $\%$ & $\mathbf{F}$ & $\%$ & $F$ & $\%$ & $\mathbf{F}$ & $\%$ & $\mathbf{F}$ & $\%$ \\
\hline $\begin{array}{l}\text { ¿En qué grado el acceso al servicio público } \\
\text { de educación se ha visto afectado econó- } \\
\text { micamente? }\end{array}$ & 1 & 4,5 & 0 & 0,0 & 0 & 0,0 & 9 & 40,9 & 12 & 54,5 & 22 & 100 \\
\hline $\begin{array}{l}\text { ¿Qué tan efectiva considera usted a la edu- } \\
\text { cación virtual en el proceso educativo? }\end{array}$ & 4 & 18,2 & 2 & 9,1 & 12 & 54,5 & 4 & 18,2 & & 0,0 & 22 & 100 \\
\hline $\begin{array}{l}\text { ¿En qué medida mejoraría el proceso edu- } \\
\text { cativo del estudiante si se implementa una } \\
\text { estrategia didáctica dentro del proceso de } \\
\text { la educación virtual en tiempos de pande- } \\
\text { mia? }\end{array}$ & 0 & 0,0 & 0 & 0,0 & 2 & 9,1 & 16 & 72,7 & 4 & 18,2 & 22 & 100 \\
\hline
\end{tabular}

Nota. Escala de 1 a 5 , donde $1=$ nada, 2 = bajo, 3 = moderado, $4=$ alto, $5=$ muy alto, $f=$ frecuencia $y \%=$ porcentaje 
Además, se aplicaron preguntas abiertas:

- ¿Cuál considera Usted el principal impacto de la pandemia sobre el acceso al servicio público de educación en la ciudad de Tulcán? Por qué.

- ¿En qué grado valora Usted como docente la necesidad de nuevas estrategias didácticas para el mejoramiento del proceso de enseñanza - aprendizaje en tiempos de pandemia?

- ¿Podría Usted dar tres sugerencias para ser tomadas en cuenta en la elaboración de una estrategia didáctica como una propuesta para el mejoramiento del proceso enseñanza-aprendizaje en la educación en tiempos de pandemia?

Los principales resultados se visualizan en las siguientes tablas:

Tabla 2. Principales impactos de la pandemia sobre el acceso al servicio público de educación en la ciudad de Tulcán.

Fuente: elaboración propia basada en las encuestas a docentes

1 Impacto económico, por egresos no presupuestados para herramientas tecnológicas

2 Deserción escolar

3 Perdida de la universalidad de la educación, por la brechas económico-social

4 La falta de herramientas tecnológicas a bajo costo o subsidiada por parte del Estado dificultan la conectividad para la modalidad de educación online

5 Falencias en el uso Tecnologías de información y comunicación (TIC)

6 Disminución de la calidad educativa en todos los estudiantes

7 Aumento de las horas de trabajo docente.

Tabla 3. Sugerencias para la elaboración de una estrategia didáctica como una propuesta para el mejoramiento del proceso enseñanzaaprendizaje en la educación en tiempos de pandemia. Fuente: elaboración propia basada en las encuestas a docentes

$1 \quad$ Los docentes juntamente con el DECE deben evaluar las necesidades académicas y emocionales de los estudiantes.

2 Evaluar, mejorar y desarrollar un sistema de comunicación efectivo entre todos los miembros de la comunidad educativa.

Establecer una correcta secuencia didáctica basada en los referentes curriculares actuales y las necesidades actuales de los

3 estudiantes en los cuales se indiquen cuatro etapas de la secuencia didáctica como la de comprensión, descontextualización, resolución de la situación problema y etapa de reflexión.

4 Se lograría un correcto aprendizaje realizando: apertura de la unidad, saberes previos, conocimiento, análisis, ejemplos, actividades de aprendizaje y evaluación.

$5 \quad$ Motivar la lectura mediante la presentación de casos prácticos de estudio y que tengan trascendencia en la sociedad.

6 Trabajo autónomo dinámico dirigido a los estudiantes.

$7 \quad$ Aprendizaje basado en las teorías del aprendizaje conectivista y el constructivismo social.

Tres sugerencias para elaborar "Estrategias para la enseñanza en grupo":

8

a. Emplear estructuras de comunicación de colaboración.

b. Generar mecanismos que permitan que los resultados sean siempre compartidos por el grupo.

9 Propiciar la participación de todos los miembros de forma equitativa y solidaria. 
En la presente tabla se encuentran los resultados de las encuestas aplicadas a los estudiantes de educación media y superior con respecto al impacto del COVID-19.

Tabla 4. Encuesta aplicada a estudiantes de educación media y superior.

Fuente: elaboración propia basada en las encuestas a estudiantes

\begin{tabular}{|c|c|c|c|c|c|c|c|c|c|c|c|c|}
\hline \multirow{2}{*}{ PREGUNTA } & \multicolumn{2}{|c|}{1} & \multicolumn{2}{|c|}{2} & \multicolumn{2}{|c|}{3} & \multicolumn{2}{|c|}{4} & \multicolumn{2}{|c|}{5} & \multicolumn{2}{|c|}{ Total } \\
\hline & $F$ & $\%$ & $F$ & $\%$ & $F$ & $\%$ & $\mathbf{F}$ & $\%$ & $F$ & $\%$ & $F$ & $\%$ \\
\hline $\begin{array}{l}\text { ¿Qué tan efectiva considera usted } \\
\text { a la educación virtual en el proceso } \\
\text { educativo }\end{array}$ & 0 & 0,0 & 14 & 35,0 & 14 & 35,0 & 8 & 20,0 & 4 & 10,0 & 40 & 100 \\
\hline $\begin{array}{l}\text { ¿En qué medida mejoraría el proce- } \\
\text { so de educación del estudiante si } \\
\text { se implementaran nuevas estrate- } \\
\text { gias didácticas dentro del proceso } \\
\text { de educación virtual en tiempos de } \\
\text { pandemia? }\end{array}$ & 0 & 0,0 & 1 & 2,5 & 9 & 22,5 & 22 & 55,0 & 8 & 20,0 & 40 & 100 \\
\hline $\begin{array}{l}\text { ¿En qué medida la capacidad de } \\
\text { conectividad a internet ha afectado } \\
\text { el acceso al servicio público de la } \\
\text { educación? }\end{array}$ & 0 & 0,0 & 5 & 12,5 & 8 & 20,0 & 18 & 45,0 & 9 & 22,5 & 40 & 100 \\
\hline
\end{tabular}

En la tabla siguiente se presentan los resultados obtenidos de las encuestas realizadas a los padres de familia de los estudiantes de Tulcán, con la finalidad de recopilar información sobre el impacto del COVID-19.

Tabla 5. Encuesta aplicada a padres de familia. Fuente: elaboración propia

\begin{tabular}{|c|c|c|c|c|c|c|c|c|c|c|c|c|}
\hline \multirow{2}{*}{ PREGUNTA } & \multicolumn{2}{|c|}{1} & \multicolumn{2}{|c|}{2} & \multicolumn{2}{|c|}{3} & \multicolumn{2}{|c|}{4} & \multicolumn{2}{|c|}{5} & \multicolumn{2}{|c|}{ Total } \\
\hline & $F$ & $\%$ & $\mathbf{F}$ & $\%$ & $\mathbf{F}$ & $\%$ & $\mathbf{F}$ & $\%$ & $\mathbf{F}$ & $\%$ & $F$ & $\%$ \\
\hline $\begin{array}{l}\text { ¿En qué grado la pandemia ha } \\
\text { generado impactos sobre el ac- } \\
\text { ceso al servicio público de edu- } \\
\text { cación? }\end{array}$ & 0 & 0,0 & 2 & 8,0 & 5 & 20,0 & 6 & 24,0 & 12 & 48,0 & 25 & 100 \\
\hline $\begin{array}{l}\text { ¿En qué grado el acceso al ser- } \\
\text { vicio público de educación se ha } \\
\text { visto afectado económicamente? }\end{array}$ & 2 & 8,0 & 2 & 8,0 & 7 & 28,0 & 3 & 12,0 & 11 & 44,0 & 25 & 100 \\
\hline $\begin{array}{l}\text { ¿En qué medida la capacidad de } \\
\text { conectividad a Internet ha afec- } \\
\text { tado el acceso al servicio público } \\
\text { de la educación? }\end{array}$ & 1 & 4,0 & 4 & 16,0 & 3 & 12,0 & 6 & 24,0 & 11 & 44,0 & 25 & 100 \\
\hline
\end{tabular}




\begin{tabular}{|c|c|c|c|c|c|c|c|c|c|c|c|c|}
\hline $\begin{array}{l}\text { ¿En qué medida mejoraría el pro- } \\
\text { ceso de enseñanza-aprendizaje } \\
\text { del estudiante si se implementa- } \\
\text { ran nuevas estrategias didácti- } \\
\text { cas dentro del proceso de la edu- } \\
\text { cación en tiempos de pandemia? }\end{array}$ & 2 & 8,0 & 2 & 8,0 & 5 & 20,0 & 11 & 44,0 & 5 & 20,0 & 25 & 100 \\
\hline $\begin{array}{l}\text { ¿En qué grado el Estado ecuato- } \\
\text { riano ha garantizado el acceso al } \\
\text { servicio público de educación? }\end{array}$ & 6 & 24,0 & 5 & 20,0 & 10 & 40,0 & 4 & 16,0 & 0 & 0,0 & 25 & 100 \\
\hline
\end{tabular}

Nota. Escala de 1 a 5, donde 1 = nada, 2 = bajo, 3 = moderado, $4=$ alto, $5=$ muy alto, $f=$ frecuencia y $\%=$ porcentaje

En las siguientes tablas se presentan los resultados de las encuestas aplicada a abogados, con la finalidad de determinar el impacto, los derechos, los preceptos, y políticas públicas, generados por la pandemia COVID 19 en Tulcán.

Tabla 6. Encuesta aplicada a abogados. Fuente: elaboración propia

\begin{tabular}{|c|c|c|c|c|c|c|c|c|c|c|c|c|}
\hline \multirow{2}{*}{ PREGUNTA } & \multicolumn{2}{|c|}{1} & \multicolumn{2}{|c|}{2} & \multicolumn{2}{|c|}{3} & \multicolumn{2}{|c|}{4} & \multicolumn{2}{|c|}{5} & \multicolumn{2}{|c|}{ Total } \\
\hline & $\mathbf{F}$ & $\%$ & $F$ & $\%$ & $\mathbf{F}$ & $\%$ & $\mathbf{F}$ & $\%$ & $F$ & $\%$ & $F$ & $\%$ \\
\hline $\begin{array}{l}\text { Al ser la educación un de- } \\
\text { recho humano según el en- } \\
\text { foque del Buen Vivir, con la } \\
\text { pandemia ¿en qué grado se } \\
\text { ha visto vulnerado? }\end{array}$ & 0 & 0,0 & 2 & 7,7 & 2 & 7,7 & 12 & 46,2 & 10 & 38,5 & 26 & 100 \\
\hline $\begin{array}{l}\text { En tiempos de pandemia } \\
\text { ¿en qué medida se ha efec- } \\
\text { tivizado el acceso al servicio } \\
\text { público de educación con } \\
\text { base en los derechos del } \\
\text { buen vivir? }\end{array}$ & 6 & 23,1 & 6 & 23,1 & 10 & 38,5 & 2 & 7,7 & 2 & 7,7 & 26 & 100 \\
\hline $\begin{array}{l}\text { ¿En qué medida la falta de } \\
\text { acceso a medios tecnológi- } \\
\text { cos, el desconocimientode } \\
\text { herramientas tecnológicas, } \\
\text { y la baja conectividad de } \\
\text { docentes y estudiantes, han } \\
\text { afectado el acceso al ser- } \\
\text { vicio público de educación } \\
\text { con base en los derechos } \\
\text { del buen vivir? }\end{array}$ & 0 & 0,0 & 2 & 7,7 & 4 & 15,4 & 6 & 23,1 & 14 & 53,8 & 26 & 100 \\
\hline
\end{tabular}




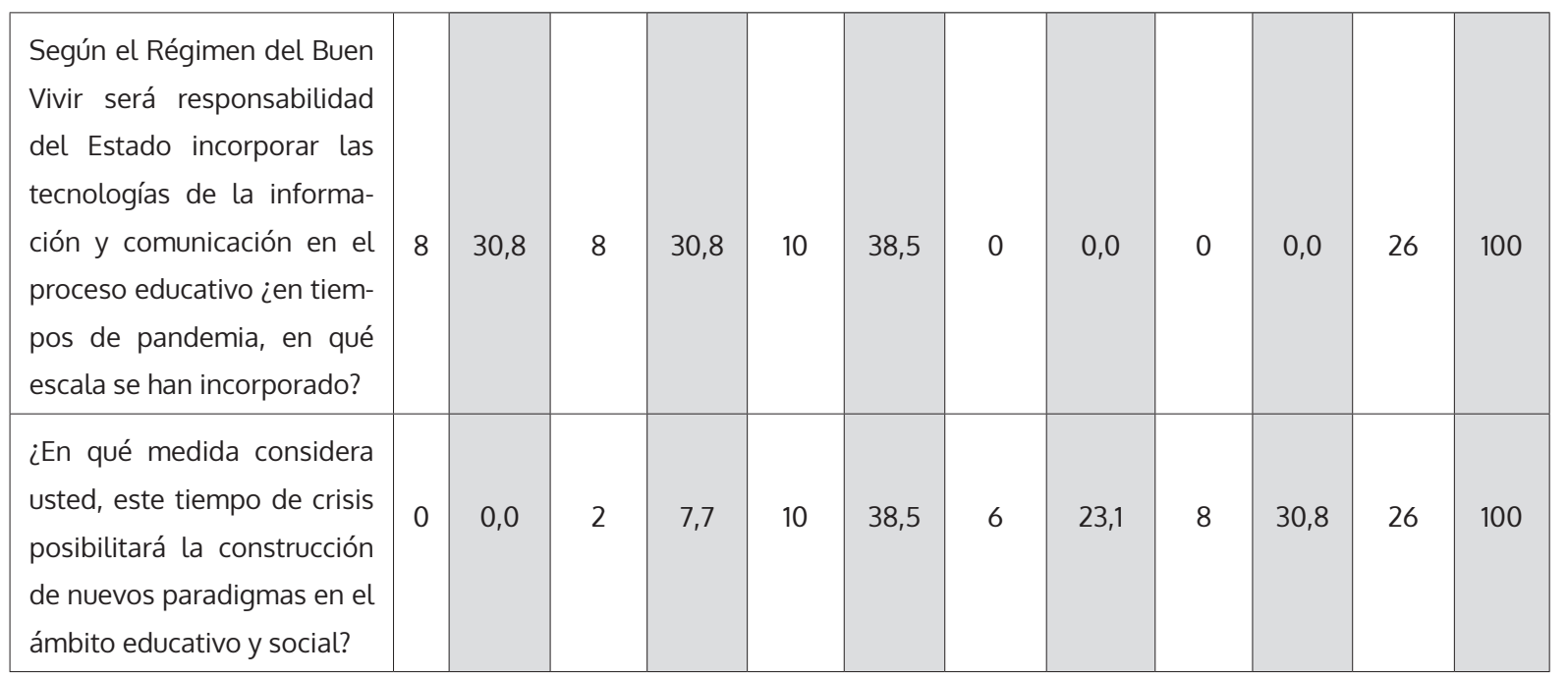

Nota. Escala de 1 a 5, donde 1 = nada, 2 = bajo, $3=$ moderado, $4=$ alto, $5=$ muy alto, $f=$ frecuencia y $\%=$ porcentaje

Tabla 7. Principales impactos jurídicos de la pandemia sobre el acceso al servicio público de educación con base en los derechos del buen vivir establecidos en la Constitución de la República. Fuente: elaboración propia basada en las encuestas a abogados

\begin{tabular}{|c|l|}
\hline $\mathbf{1}$ & Cierre total y universal de centros educativos de todo nivel. \\
\hline $\mathbf{2}$ & Recesión económica a toda escala. \\
\hline $\mathbf{3}$ & Falta de normativa y políticas públicas para enfrentar casos futuros similares o afines. \\
\hline $\mathbf{4}$ & Conglomerado desprovisto del servicio público de educación, a falta de implementación de tecnología por el Estado. \\
\hline $\mathbf{5}$ & Reducción de salario a los educadores. \\
\hline $\mathbf{6}$ & Aumento de desigualdad y discriminación social. \\
\hline $\mathbf{7}$ & Vulneración, marginación y exclusión del derecho a la educación por brechas económico-sociales. \\
\hline $\mathbf{8}$ & Baja en la calidad de los procesos de enseñanza-aprendizaje por la virtualización de los procesos educativos \\
\hline $\mathbf{9}$ & Inaccesibilidad a centros educativos en razón al ambiente físico, psíquico y moral \\
\hline 10 & Quebrantamiento a la participación diversa, de inclusión e interculturalidad de los actores educativos \\
\hline 11 & El buen vivir en estos tiempos termina siendo una quimera \\
\hline 12 & Aumento del índice de violencia intrafamiliar \\
\hline
\end{tabular}

Tabla 8. Preceptos jurídicos que regularicen el desenvolvimiento del servicio público de educación en tiempos de pandemia, orientados a hacer efectivo el buen vivir. Fuente: elaboración propia basada en las encuestas a abogados

1 Potenciar el desarrollo de actividades ciudadanas por medios tecnológicos mediante Acuerdos Ministeriales.

2

Reformar la Ley de Educación para el fortalecimiento de espacios de participación ciudadana a través de medios tecnológicos y con las medidas sanitarias del caso. 




Tabla 9. Políticas públicas, que garanticen el acceso al servicio público de educación con base en los derechos del buen vivir. Fuente: elaboración propia basada en las encuestas a abogados

1 Instaurar programas de gobierno para hacer efectivo el acceso igualitario a la educación en tiempos de emergencia sanitaria, estados de excepción, desastres naturales, entre otros.

2 Eliminar aranceles de importación en la adquisición de equipos tecnológicos que sirvan para una educación vanguardista.

3 Establecer dentro del presupuesto general asignado a la educación un considerable porcentaje para apoyo e innovación tecnológica.

4 Fundar un proyecto de gobierno dirigido a la comunidad educativa conducente a la sustentación de una cultura digital.

5 Programa de gobierno que busque potenciar las infraestructuras públicas y privadas por medio de sistemas de información, redes y servicios de internet de gran capacidad que llegue a todos lados.

6 Instaurar incentivos a trabajadores y empleadores de la educación.

7 Reapertura de las escuelas rurales en los lugares alejados de la patria.

Instituir plan de gobierno encaminado a entregar a cada estudiante de las instituciones de educación pública una laptop a fin de que la educación esté a la vanguardia de los tiempos modernos.

Asistir con servicio de internet y computadoras que pueden ser proporcionadas en calidad de préstamo de las escuelas cercanas a las Tenencias Políticas a fin de que se convierta en el centro de educación vía online para los estudiantes que no posean este servicio en cumplimiento de las medidas de bioseguridad.

\section{DISCUSIÓN}

Con base en los resultados de las Tablas 1, 4 y 5; los docentes, estudiantes y padres de familia, manifiestan que la pandemia ha generado un impacto que va de muy alto a alto en $77,3 \%$; $82,5 \%$ y $72 \%$ respectivamente sobre el acceso al servicio público de educación; lo que evidencia que la pandemia ha incidido en el proceso educativo en un grado significativamente alto. 
Con respecto al grado de afectación económica sobre el acceso al servicio público de educación los docentes (tabla 1) y padres de familia (tabla 5), indican que ha afectado en una escala que va de muy alto a alto en $95,4 \%$ y $56 \%$ en orden; lo que se evidencia que el impacto económico es mayor en los docentes, como consecuencia de la baja salarial ejecutada dentro de la Ley Orgánica de Apoyo Humanitario e inversiones en herramientas tecnológicas que adquirieron los docentes para llevar acabo las clases online.

En cuanto a la efectividad de la educación virtual en el proceso educativo en tiempos de pandemia, los docentes (Tabla 1$)$ consideran en un grado que va de moderado a nada $(81,8 \%)$ y los estudiantes (Tabla 4) consideran que va de moderado a bajo (70\%); esto evidencia, que la educación virtual no ha sido tan efectiva en el proceso enseñanza-aprendizaje.

Con base en los resultados obtenidos en las Tablas 1, 4 y 5, los docentes señalan en un $90,9 \%$ que va de alto a muy alto el proceso educativo del estudiante mejoraría si se implementa una estrategia didáctica dentro del proceso de la educación virtual en tiempos de pandemia, igualmente opinan los estudiantes (75\%) y los padres de familia (64\%); esto hace necesario una estrategia didáctica como propuesta para el mejoramiento del proceso de enseñanza-aprendizaje para los tiempos de pandemia.

En referencia a la capacidad de conectividad al internet, esta ha afectado al acceso al servicio público de la educación, los estudiantes (tabla 4) en un 67,5\% opinan que va de alto a muy alto y los padres de familia en un $66 \%$ opinan que va desde muy alto a alto, lo que demuestra que hay limitaciones de conectividad al internet.

En lo concerniente al grado en que el Estado ecuatoriano ha garantizado el acceso al servicio público de educación en tiempos de pandemia, los padres de familia (tabla 5), en un $84 \%$ manifiestan que va de moderado a nada; esto pude deberse a la limitación económica del Estado y su injerencia en la crisis general de la sociedad.

Además, la UNESCO (2020) ha estimado que, a nivel mundial, al menos 1184126508 de estudiantes se han visto afectados directamente en su proceso de aprendizaje, pero, además, se ven perjudicados sus familiares, docentes y administrativos, y conlleva a efectos negativos en la economía de las naciones, siendo más fuerte el impacto en los pobres y necesitados (Jiménez-Sánchez, 2020).

Con base en los resultados obtenidos en las tablas 2 y 7 de encuestas aplicadas a docentes y abogados, se establecen como impactos de la pandemia sobre el acceso al servicio público de educación y los derechos del buen vivir establecidos en la Constitución de la República en la 
ciudad de Tulcán, los siguientes: educativo, económico, social, jurídico, tecnológico e impacto de aislamiento.

Es así como la pandemia de enfermedad por coronavirus (COVID-19) ha provocado una crisis sin precedentes en todos los ámbitos. En la esfera de la educación, esta emergencia ha dado lugar al cierre masivo de las actividades presenciales de instituciones educativas en más de 190 países con el fin de evitar la propagación del virus y mitigar su impacto (Equipo de Dirección, 2020).

En la Tabla 6 se presentaron los resultados de las encuestas aplicadas a los abogados, en donde indican que al ser la educación un derecho humano según el enfoque del Buen Vivir, con la pandemia este se ha visto vulnerado en un grado que va de alto a muy alto $(84,7 \%)$. Con respecto al acceso al servicio público de educación con base en los derechos del buen vivir, en tiempos de pandemia se ha efectivizado en un $84,7 \%$ que va de moderado a nada.

En lo concerniente a la falta de acceso a medios tecnológicos, el desconocimiento de herramientas tecnológicas, y la baja conectividad de docentes y estudiantes, han afectado el acceso al servicio público de educación con base en los derechos del buen vivir en 76,9\% que va en una escala de muy alto a alto. Así mismo, al establecer el Régimen del Buen Vivir que es responsabilidad del Estado incorporar las tecnologías de la información y comunicación en el proceso educativo, señalan que, en tiempos de pandemia, se han incorporado en una escala que va de moderado a bajo (100\%).

Finalmente, los abogados encuestados consideran que este tiempo de crisis posibilita la construcción de nuevos paradigmas en el ámbito educativo y social en un 92,3\%, en una escala que va de moderado a muy alto.

Como puede inferirse en el análisis precedente, la educación es un derecho humano intrínseco y un medio indispensable para realizar otros derechos humanos, que acorde a las disposiciones constitucionales es un derecho que debe ser garantizado y ejercido por todas las personas en cualquier nivel, a través de un sistema de educación incluyente y diversa, conforme el régimen del Buen Vivir que plantea un modelo educativo inclusivo y universal (Erazo, 2020).

Derecho que en las últimas décadas se ha visto vulnerado, porque el mundo ha sido testigo de un número creciente de crisis ocasionadas por conflictos, desastres naturales y epidemias que obligan a tecnificar el proceso educativo, pero con una barrera urgente en Ecuador, pues solo el 37\% de los hogares tiene conexión a internet, lo que significa que 6 de cada 10 niños no pueden continuar sus estudios a través de plataformas digitales, la situación es más 
grave para los niños de zonas rurales, donde sólo el 16\% de los hogares tiene conectividad (UNICEF, 2020).

Al estar en estos tiempos de pandemia frente a una eminente virtualización de la educación los derechos se deben modernizar e incorporar, al efecto se coincide plenamente con Claudia Uribe directora de la Oficina Regional de Educación de la UNESCO para América Latina y el Caribe cuando manifiesta que "la conectividad debería ser un derecho humano". Además, la UNESCO (2020) revalida la posibilidad de la construcción de nuevos paradigmas en el ámbito educativo y social como resultado de esta crisis, cuando manifiesta que la educación en estos tiempos permite la transformación de los educandos y a la vez estos de las sociedades, sin desaprovechar esta oportunidad para cambiar el aprendizaje y construir un mundo mejor.

Ahora bien, al aparecer el Derecho como un orden social y un sistema de normas jurídicas que regulan la conducta de los hombres, estas constituyen el objeto de la ciencia del derecho, pero a la vez las ciencias de la naturaleza recurren a las leyes para explicar los fenómenos naturales, en donde los actos de la humanidad se explican con el Derecho a través de normas jurídicas (Kelsen, 2009); lo que infiere que, en estos tiempos de pandemia el Estado ecuatoriano $\mathrm{y}$ todos los Estados del mundo se han visto obligados en adoptar un sinnúmero de preceptos jurídicos, en todos los ámbitos, pero el que ocupa el campo educativo en su desenvolvimiento como servicio público, orientado a hacer efectivo el buen vivir.

Es así como, en Ecuador, según INEN (2020) establece un índice de legislación en emergencia sanitaria en el que detalla decretos ejecutivos, acuerdos ministeriales, acuerdos interministeriales, resoluciones, pero también a lo que se suma la expedición de leyes como la Ley Orgánica de Apoyo Humanitario; asimismo a nivel internacional han surgido varios mandatos normativos por ejemplificar la Resolución 1/2020 de la Comisión Interamericana de Derechos Humanos, que dispone en cuanto al derecho a la educación, que los Estados deben disponer de mecanismos que permitan el acceso a la educación y con estímulos de edad y nivel de desarrollo (CIDH, 2020); pues, el buen vivir en su constructo se encuentra basado en el goce efectivo de los derechos, armonía con la naturaleza, bien común e interés general, y democracia (León, 2015). Lo que se revalida con base en los resultados obtenidos en la tabla 8 de las encuestas aplicadas a abogados y adhiere la posibilidad de reformas a normas especiales, expedición de leyes requirentes, así como el establecimiento de políticas públicas.

La Constitución de la República del Ecuador del año 2008 incorporó por primera vez la noción del Buen Vivir, noción que ha evolucionado y hoy por hoy se constituye en un concepto multidimensional, incorporando métricas como la educación, salud, vivienda, entre otras (de- 
rechos del buen vivir), que buscan contribuir con indicadores que guíen la formulación de políticas públicas (León, 2015), mismas que son el reflejo de los ideales y deseos de la colectividad, enuncian los objetivos de bienestar colectivo y permiten entender hacia dónde se quiere orientar el desarrollo y cómo hacerlo, evidenciando lo que se procura conseguir con la intervención pública y cómo se distribuyen las responsabilidades y recursos entre los actores sociales (Torres \& Santander, 2013), reguladas constitucionalmente en el artículo 85 para su formulación, ejecución, evaluación y control (Asamblea Constituyente República del Ecuador, 2008).

Justamente, en los resultados obtenidos en la tabla 9 de encuestas aplicadas a abogados se establecen políticas públicas, que garanticen el acceso al servicio público de educación con base en los derechos del buen vivir, políticas con influencia en las esferas incidentes en la educación como: casos fortuitos o fuerza mayor, accesibilidad tecnológica, económico-tributaria, presupuestarias y emplazamiento geográfico.

\subsection{Guía para emprender la transformación de la educación}

Se propone como estrategia didáctica el reforzar la práctica de la teoría conectivista y la implementación de una guía de aprendizaje, como herramientas que permitan solventar las limitaciones a las distancias transaccionales, entre docente-estudiante, lo que facilita el desarrollo de la autonomía, responsabilidad y compromiso, de los actores educativos.

El Conectivismo, como teoría del conocimiento es creada por George Siemens, Stephen Downes y comienza a difundirse a partir del año 2004; cuya base doctrinal se fundamenta en la premisa de que aprendemos haciendo conexiones con otras personas, conceptos e ideas. Dicho conocimiento según los autores es una red y se construye como tal, el aprendizaje se basa en nuestra capacidad para formar esas conexiones y construir dichas redes (Solorzano y García, 2016).

La gestión del conocimiento a través de los nuevos procesos de comunicación que son posibles gracias al uso de redes tecnológicas y los nuevos canales de información es actualmente la materia prima o sustancia base de muchos procesos de formación a distancia, por lo que se constituye en el recurso estratégico del desarrollo actual y se coloca en el centro de los procesos de transformación social.

Con estos importantes aportes de utilizar las TIC como medio de comunicación y educación, se debe hacer especial referencia a los entornos personales de aprendizaje (PLE); como gestores del aprendizaje y se encuentran como servicios en la web, instrumentos que deben ser aprovechados por parte del Ministerio de Educación o las diferentes Unidades Educativas 
para comunicar eficientemente la educación, utilizando la conectividad de los servicios virtuales, rompiendo barreras institucionales y permitiendo la universalización del conocimiento. Los PLE retoman las ideas vigotskianas de aprendizaje y constructivismo social y las recrea en las ideas conectivistas de Siemens y Downes para la era de Internet (Solorzano y García, 2016). El segundo elemento fundamental que se considera en la propuesta es la elaboración de las guías de aprendizaje por parte del ministerio rector. A decir de la Real Academia Española (RAE), una guía es aquello que dirige o encamina y, en este sentido, la guía de aprendizaje sistematiza las mediaciones o intervenciones que los docentes desean realizar para orientar el desarrollo de las tareas que afrontan los estudiantes en su proceso de enseñanza aprendizaje. Por tanto, las mediciones que involucra la guía de aprendizaje que se desea definir son de carácter escrito (Londoño, 2017).

La emisión de este importante documento permite sintetizar los conocimientos priorizados que van a ser objeto de conocimiento del estudiante en base al currículo general, proporciona una función orientadora e integradora al direccionar la organización y sistematización del aprendizaje, con una acción motivadora y dialógica que incentive permanentemente a sus actores. Lo que se propone mediante ello es facilitar de manera integral el aporte interdisciplinario de cada docente en los diferentes ámbitos del saber humano, permitiendo sintetizar los objetivos, destrezas y fines de la educación, promoviendo la reflexión y retroalimentación del estudiante.

Se ha visto pertinente considerar la presente propuesta, como una posible alternativa de solución del problema expuesto en el presente trabajo, estas medidas permiten de cierta manera superar las limitaciones que actualmente se imponen a la labor educativa.

\section{CONCLUSIONES}

Mediante procesos de análisis de los resultados, se determinó que el impacto de la pandemia sobre el acceso al servicio público de educación, al igual que la vulneración de la educación como derecho humano según el enfoque del buen vivir van en un grado ascendente de alto a muy alto, lo que razonablemente confirma una efectivización en escala que va de moderado a nada sobre el acceso al servicio público de educación con base en los derechos del buen vivir en tiempos de pandemia.

En tiempos de pandemia se hace necesario la implementación de propuestas, como es el caso de la guía para emprender la transformación de la educación, que permita la superación 
de las actuales condiciones que están perjudicando al proceso de enseñanza-aprendizaje, en su efectividad, calidad y calidez.

El presente paper tuvo como limitaciones que por tiempos de pandemia se estableció una muestra no probabilística y tomada por conveniencia, además los estudiantes y docentes no se encontraron concentrados en sus instituciones educativas de trabajo, por esta razón, la recopilación de los datos no se la realizó personalmente y así evitar el contagio del COVID-19.

Como trabajo futuro, se puede expandir este estudio a los nuevos procesos de enseñanzaaprendizaje que se darán después de la pandemia; es decir, cuando todos los estudiantes y docentes se integren nuevamente a sus labores dentro de las instituciones educativas; puesto que posiblemente se generarán nuevos impactos en el período de post pandemia en el sector educativo.

\section{REFERENCIAS BIBLIOGRÁFICAS}

Asamblea Constituyente República del Ecuador. (2008, 20 de octubre). Constitución de la República del Ecuador. Registro Oficial N. 449. https://www.wipo.int/edocs/lexdocs/laws/es/ec/ec030es.pdf

Comisión Interamericana de Derechos Humanos. (2020, 10 de abril). Resolución No. 1/2020. Pandemia $y$ derechos humanos en las américas. https://www.oas.org/es/cidh/decisiones/pdf/Resolucion1-20-es.pdf

Equipo de Dirección. (2020). Geopolítica de la pandemia de COVID-19. Geopolítica(s), 11, 11-13. https:// doi.org/10.5209/GEOP.69137

Erazo, M. (2020, 14 de mayo). Las obligaciones en salud y educación en cuarentena. INREDH. https:// inredh.org/las-obligaciones-en-salud-y-educacion-en-cuarentena/

Gómez Armijos, C. et al. (2017). La Investigación Científica y las Formas de Titulación: Aspectos Conceptuales y prácticos. Editorial Jurídica del Ecuador.

Hernández-Sampieri, R., y Mendoza Torres, Ch. (2018). Metodología de la Investigación. Las rutas cuantitativa, cualitativa y mixta. McGRAW-HILL Interamericana Editores, S.A. de C. V.

INEC (2020, abril). Índice de legislación abril 2020 emergencia sanitaria. https://www.ecuadorencifras. gob.ec/documentos/web-inec/Resoluciones/2020/Indice legislacion 2020/\%C3\%8Dndice abril_emergencia.pdf 
Jiménez-Sánchez, C. (2020). Impacto de la Pandemia por SARS-CoV2 sobre la Educación. Revista Electrónica Educare, 24, 1-3. https://dialnet.unirioja.es/servlet/articulo?codigo=7538299

Kelsen, H. (2009). Teoría Pura del Derecho. Editorial Universitaria de Buenos Aires. (Original publicado en 1960). http://www.herrerapenaloza.com/images/biblioteca/Teoria\%20pura\%20del\%20derecho.\%20Hans\%20kelsen.pdf

Latapí, P. (2009). El derecho a la educación: su alcance, exigibilidad y relevancia para la política educativa. Revista Mexicana de Investigación Educativa, 14(40), 255- 287. https://www.redalyc.org/ articulo.oa?id=14004012

León, M. (2015). Del discurso a la medición: Propuesta metodológica para medir el Buen Vivir en Ecuador. Libro metodológico del Instituto Nacional de Estadística y Censos (INEC). https:// www.google.com/url?sa=t\&rct=j\&q=\&esrc=s\&source=web\&cd=\&ved=2ahUKEwjDxe7fhaf wAhUpTd8KHZxvDfsQFjAAegQIAhAD\&url=https\%3A\%2F\%2Fwww.ecuadorencifras.gob. ec\%2Fwp-content\%2Fuploads\%2Fdownloads\%2F2016\%2F10\%2FBuen-Vivir-en-el-Ecuador. pdf\&usg=AOvVaw2mBuxAzZIjK6-BUw26nJeC

Londoño, Y. (2017). Documento orientador para la elaboración de guías de aprendizaje. Mineducación.

Scioscioli, S. (2016). El derecho a la educación como derecho fundamental y sus alcances en el derecho internacional de los derechos humanos. Fournal of Supranational Policies of Education, 2, 6-24. https://revistas.uam.es/index.php/jospoe/article/view/5625

Solorzano, F. y García, A. (2016). Fundamentos del Aprendizaje en Red desde el Conectivismo y la Teoría de la Actividad. Revista Cubana de Educación Superior, (3), 98-112. http://scielo.sld.cu/ pdf/rces/v35n3/rces08316.pdf

Torres, J. y Santander, J. (2013). Introducción a las políticas públicas:

conceptos y herramientas desde la relación entre Estado y ciudadanía. IEMP Ediciones. https://www. funcionpublica.gov.co/eva/admon/files/empresas/ZW1 wcmVzYV83Ng==/imgproductos/1450056996 ce38e6d218235ac89d6c8a14907a5a9c.pdf

UNESCO (2020, 18 de junio). Reconstruir mejor: tras el COVID-19, la educación debe cambiar para responder a la crisis climática. https://es.unesco.org/news/reconstruir-mejor-covid-19-educaciondebe-cambiar-responder-crisis-climatica

UNICEF (2020). Educación. https://www.unicef.org/ecuador/s\%C3\%BAmate-la-respuesta-ante-el-coronavirus

UNICEF (2020, 4 de marzo). El nuevo coronavirus y el derecho a la educación. https://www.unicef.es/ educa/blog/nuevo-coronavirus-derecho-educacion 